\title{
Low/Hard State Spectra of Neutron Star X-ray Binary 4U 1700+24 Observed with Suzaku and XMM - Newton
}

\author{
Osamu Nagae, Hiromitsu Takahashi, Hirohisa Shirai and Yasushi Fukazawa \\ Department of Physical Science, School of Science, Hiroshima University, Japan \\ E-mail: nagae@hep01.hepl.hiroshima-u.ac.jp
}

\begin{abstract}
We present the spectral analysis of Suzaku and XMM - Newton observations of the neutron star (NS) X-ray binary, $4 \mathrm{U} 1700+24$. These observation data were obtained in 2002 August, 2003 March, 2003 August (XMM - Newton), and 2007 August (Suzaku). The 0.3-20 keV X-ray luminosity observed with Suzaku is $\sim 1.1 \times 10^{32} \mathrm{erg} / \mathrm{s}$ corresponding to $\sim 10^{-6} L_{\mathrm{Edd}}$, where $L_{\mathrm{Edd}}$ is Eddington luminosity. The X-ray luminosity observed with $X M M-$ Newton is $\sim 10^{-4}-10^{-5}$ $L_{\mathrm{Edd}}$ in $0.3-10 \mathrm{keV}$. The distance to the source is so close, $420 \mathrm{pc}$, that we can obtain the X-ray energy spectrum even at such an extremely low luminosity. Both Suzaku and XMM-Newton spectra above $\sim 1 \mathrm{keV}$ can be explained by a Comptonized blackbody which is thought to be arisen from NS surface. Moreover, we detect a soft excess below $1 \mathrm{keV}$. The softer component is thought to be Comptonized radiation coming from the accretion disk or accretion flow. From the observed flux ratio of each component, the radiative efficiency of the accretion disk of $4 \mathrm{U}$ $1700+24$ is $\sim 10$ times larger than that of Sgr A*.
\end{abstract}

VII Microquasar Workshop: Microquasars and Beyond

September 1 - 5, 2008

Foca, Izmir, Turkey 


\section{Introduction}

Low mass X-ray binaries (LMXBs) contain a neutron star (hereafter NS-LMXB) or a blackhole (hereafter BH-LMXB), where matter accrete from a low mass $\left(\sim 1 M_{\odot}\right)$ main-sequence star or a slightly evolved late-type star. X-ray spectra of LMXBs exhibit several spectral states which depend on the luminosity (i.e., mass accretion rate $\dot{M}$ ). When their luminosities are below $\sim$ $10^{-2} L_{\text {Edd }}$, LMXBs are classified as low/hard state (LHS). For BH-LMXBs, the X-ray spectra of LHS are dominated by a hard power-law (PL) component with a photon index $\sim 1.7$ ([10]). The PL radiation is thought to be a result of Comptonized component (Comptonized thermal radiation and/or Synchrotron self Compton), although the origin and physical parameters of the Compton cloud are not well understood. The LHS spectra of NS-LMXBs are similar to those of BH-LMXBs in their LHS except for an additional soft thermal component, suggesting that the origin of the soft component is thermal emission from the NSs surface (e.g., [2]). Although there are many observations of LMXBs with the luminosities of $10^{-2}-10^{-3} L_{\mathrm{Edd}}$, observation of an extremely low luminosity ( $\lesssim 10^{-4} L_{\mathrm{Edd}}$ ) of LMXBs are poor. The purpose of this work is to learn the spectra and the accretion picture of LMXB at such an extremely low luminosity.

The number of NS-LMXBs are $\sim 10$ times larger than that of BH-LMXBs ([8]; [7]) so that NS-LMXBs are suitable to study various luminous states $\left(L_{\mathrm{Edd}}-10^{-6} L_{\mathrm{Edd}}\right.$ or may be less). Thus, NS-LMXBs are excellent laboratories to study low luminous LMXBs. Here, we focus on $4 \mathrm{U}$ $1700+24$ which contains probably NS orbiting a M-type giant star and locates at $\sim 420 \mathrm{pc}$ from the Earth ([9]; [4]). This is the nearest X-ray binary to date ([8]; [7]) so that we can observe the object even at an extremely low luminosity. In this paper, we present spectral analysis of Suzaku data $(0.3-20 \mathrm{keV})$ and archive $X M M-$ Newton data $(0.3-10.0 \mathrm{keV})$.

\section{Observation and analysis}

We observed 4U 1700+24 with Suzaku on 2007 August 22. The data were acquired with the X-ray Imaging Spectrometer (XIS) and the Hard X-ray Detector (HXD). The HXD consists of two detectors, Si PIN photo diodes observing 10-70 keV (hereafter PIN) and GSO scintillators observing 40-600 keV. For the data analysis, we used cleaned events of the XIS and the PIN distributed by Suzaku Data Processing version 2.0. We generated response files using the tools "xisrmfgen" and "xissimarfgen" available in the HEASOFT version 6.4 for the XIS data analysis. The response file of the PIN data distributed by HXD team. The non-X-ray background event is also provided by the HXD team ("PINUDLCUNIT" and version "2.0pre20071002"). The contribution of the cosmic X-ray background (CXB) was estimated with the PIN response for a point source observed at the HXD nominal position assuming the flux of CXB as $8.0 \times 10^{-4} \times(E / 1 \mathrm{keV})^{-1.29}$ $\times \exp (-E / 40 \mathrm{keV})$ photons $/ \mathrm{cm}^{2} / \mathrm{s} / \mathrm{deg}^{2} / \mathrm{keV}$ where $E$ is photon energy. The PIN spectra above $\sim$ $30 \mathrm{keV}$ is consistent with the CXB flux.

The XMM - Newton X-ray optics consist of five X-ray CCD detectors which observe simultaneously. The energy range of CCDs are $0.5-15 \mathrm{keV}$, two MOS and one PN types, comprising the European Photon Imaging Camera. The other two detectors are Reflection Grating Spectrometers (RGS) which disperse photons in the range 5-35 A, although we do not use the RGS data. There are four archival data of $4 \mathrm{U} 1700+24$ obtained on 2002 August 11, 2003 March 
7, 9, and 2003 August 13. Since the flux of the 2003 March 7 data is almost same with that of the 2003 August 13 data, we will show the results of three data (Newton A, Newton B, and Newton C, chronologically) except for the result of 2003 March 7. All data were processed using $X M M$ - Newton Science Analysis System version 8.0.0. Some data sets of the MOS are affected by pile-up, while there is no pile up in the PN data sets. Therefore, only the PN data sets are utilized.

\section{Results}

We analyzed the time averaged Suzaku spectrum to examine the averaged behavior. The X-ray luminosity at $0.3-20.0 \mathrm{keV}$ is $\sim 1.1 \times 10^{32} \mathrm{erg} / \mathrm{s}$ corresponding to $\sim 10^{-6} L_{\text {Edd. }}$. The XISO and XIS3 spectra are combined to improve the data statistics. The absorption is fixed to the Galactic value of $N_{\mathrm{H}}=4 \times 10^{20} \mathrm{~cm}^{-2}$ [3]. At first we start the analysis using a black body (BB) arisen from NS surface, but the fitting is unacceptable due to large excesses at the higher and lower energy bands. The residual is shown in Fig 1 (a). LHS spectra of NS-LMXBs is typically fitted by a Comptonized BB radiation [5]. Thus, we fit the spectrum using compPS model [14], denoted as Comp-BB, with the Comptonizing electron temperature fixed to $100 \mathrm{keV}$. Although this model is acceptable above $\sim 1 \mathrm{keV}$, it still shows large soft excess below $\sim 1 \mathrm{keV}$ (residual (b) of Fig 1). The soft excess of $4 \mathrm{U} 1700+24$ was also detected previously [15], wherein the authors added a broad $(\sigma \sim 0.1 \mathrm{keV})$ Gaussian line centered at $\sim 0.5 \mathrm{keV}$, although they mentioned that the interpretation of the broad Gaussian line is problematic. Alternatively, we try the following two modelings of the soft excess.

First, to represent the soft excess, we additionally utilize Comptonized disk black body (Comp$\mathrm{DBB}$ ) of which the seed photons are arisen from accretion disk. In contrast to the Comp-BB, the Comp-DBB assumes the seed photons come from the optically thick cool accretion disk. The residual (c) of Fig 1 shows the fitting result with the Comp-BB plus Comp-DBB models (hereafter W-CompPS), where the spectrum below $1 \mathrm{keV}$ (soft excess) can be represented by the Comp-DBB and that above $1 \mathrm{keV}$ is also represented by the Comp-BB. The obtained parameters of the $\mathrm{W}$ CompPS model are listed in Table 1. For the DBB radiation, $T_{\text {in }}$ is proportional to $L_{\text {disk }}^{1 / 4}\left(L_{\text {disk }}\right.$ is the accretion disk luminosity assuming the constant radius). Generally, $T_{\text {in }}$ is about $\sim 1 \mathrm{keV}$ when the luminosity of NS-LMXBs is close to $\sim L_{\text {Edd }}$ so that the expected value of $T_{\text {in }}$ of $4 \mathrm{U} 1700+24$ ( $\left.10^{-6} L_{\mathrm{Edd}}\right)$ is $\sim 0.01 \mathrm{keV}$ order and consistent with the obtained value, $T_{\text {in }} \lesssim 0.05 \mathrm{keV}$. Thus, we suggest that the Comp-DBB is the candidate for the origin of the soft excess.

Second, in the case of the BH-LMXBs, the advection-dominant accretion fbw (ADAF) model [13] predicts that Comptonization of synchrotron photons contribute in X-ray region. The spectra can be fitted by a simple PL with a photon index of typically $\sim 2$ (e.g., [6]). We also fit the observed spectrum of $4 \mathrm{U} 1700+24$ using the PL (instead of the Comp-DBB) plus the Comp-BB, which is also well consistent with the spectrum (see residual (d) of Fig 1). The PL index is $\sim 2.7$ and the obtained parameters of the Comp-BB are similar to those of W-CompPS. Thus, the Comptonized synchrotron radiation is also the candidate for the origin of the soft excess.

Using same models (the W-CompPS or the Comp-BB plus PL), we fitted the XMM-Newton spectra, which are roughly well fitting except for two line-like excess between $0.5-0.7 \mathrm{keV}$. The excess is represented by two narrow ( $\sigma \sim 0-0.02$ ) Gaussian centered at $\sim 0.5 \mathrm{keV}$ and $\sim 0.65 \mathrm{keV}$. However, we cannot constrain the origin of these components. 


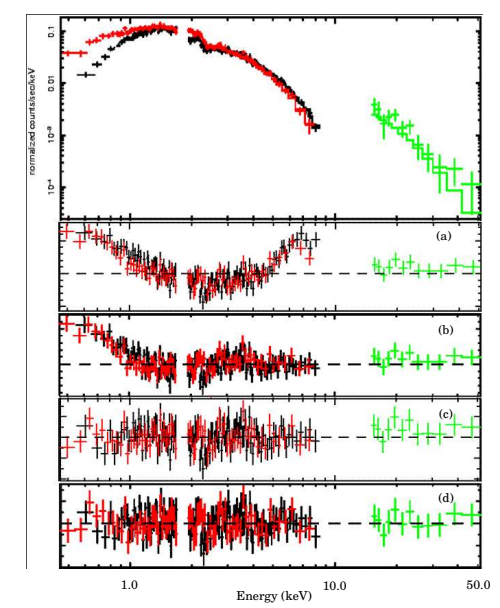

Figure 1: The XIS and PIN spectra (top panel). Panels from second to bottom show the residuals with respect to the $\mathrm{BB}$, Comp-BB, W-CompPS, and Comp-BB plus PL, respectively.

Table 1: The obtained model parameters of the W-CompPS and the Comp-BB plus PL.

\begin{tabular}{|c|c|c|c|c|c|c|c|}
\hline $\begin{array}{l}\text { Data } \\
L_{\text {Edd }}^{a} \\
\end{array}$ & Model & $\begin{array}{c}T_{\mathrm{BB}}^{b}(\mathrm{keV}) \\
T_{\mathrm{in}}^{b}(\mathrm{keV}) \\
\end{array}$ & $\begin{array}{c}\tau_{\mathrm{BB}}^{c} \\
\tau_{\mathrm{DBB}}^{c} \\
\end{array}$ & $\begin{array}{c}R_{\mathrm{BB}}^{d}(\mathrm{~m}) \\
R_{\mathrm{DBB}}^{d}(\mathrm{~km})\end{array}$ & $\mathrm{PL}^{e}$ & $\mathrm{FR}^{f}$ & $\chi^{2} /$ d.o.f \\
\hline Suzaku & W-CompPS & $0.76 \pm 0.01$ & $0.33 \pm 0.05$ & $45_{-3}^{+4}$ & & 0.28 & 1.0 \\
\hline \multirow[t]{2}{*}{$1 \times 10^{-6}$} & & $\lesssim 0.05$ & $\lesssim 0.35$ & $\gtrsim 4.2$ & & & \\
\hline & Comp-BB + PL & $0.77_{-0.03}^{+0.02}$ & $0.32 \pm 0.06$ & $45_{-4}^{+1}$ & $2.69 \pm 0.35$ & 0.17 & 1.0 \\
\hline Newton A & W-CompPS & $1.07 \pm 0.01$ & $1.71 \pm 0.03$ & $239 \pm 1$ & & 0.12 & 1.1 \\
\hline \multirow[t]{2}{*}{$9 \times 10^{-5}$} & & $0.07 \pm 0.01$ & $0.46 \pm 0.01$ & $13.3_{-0.2}^{+0.1}$ & & & \\
\hline & Comp-BB + PL & $1.06 \pm 0.01$ & $1.73_{-0.04}^{+0.03}$ & $246 \pm 1$ & $2.28_{-0.03}^{+0.02}$ & 0.11 & 1.2 \\
\hline \multirow{3}{*}{$\begin{array}{l}\text { Newton B } \\
3 \times 10^{-5}\end{array}$} & W-CompPS & $1.01_{-0.04}^{+0.01}$ & $1.27_{-0.02}^{+0.06}$ & $143_{-5}^{+11}$ & & 0.17 & 1.2 \\
\hline & & $0.06 \pm 0.01$ & $0.59_{-0.06}^{+0.10}$ & $9.3_{-0.2}^{+1.3}$ & & & \\
\hline & Comp-BB + PL & $1.00 \pm 0.03$ & $1.28_{-0.03}^{+0.04}$ & $142_{-7}^{+11}$ & $2.06_{-0.09}^{+0.07}$ & 0.18 & 1.2 \\
\hline \multirow{3}{*}{$\begin{array}{l}\text { Newton } \mathrm{C} \\
4 \times 10^{-5}\end{array}$} & W-CompPS & $0.99 \pm 0.01$ & $1.46_{-0.04}^{+0.02}$ & $165_{-5}^{+6}$ & & 0.15 & 1.3 \\
\hline & & $0.06 \pm 0.01$ & $0.42_{-0.01}^{+0.02}$ & $10.1_{-0.5}^{+0.2}$ & & & \\
\hline & Comp-BB + PL & $0.98 \pm 0.01$ & $1.45_{-0.05}^{+0.04}$ & $165_{-5}^{+1}$ & $2.32_{-0.08}^{+0.01}$ & 0.14 & 1.3 \\
\hline
\end{tabular}

${ }^{a}$ The luminosities assuming the distance is $420 \mathrm{pc} .{ }^{b} T_{\mathrm{BB}}$ and $T_{\mathrm{in}}$ are the seed-photon temperatures of the $\mathrm{BB}$ and the innermost radius of the accretion disk, respectively. ${ }^{c} \tau_{\mathrm{BB}}$ and $\tau_{\mathrm{DBB}}$ are optical depth of the Compton cloud for the BB and the $\mathrm{DBB}$, respectively. ${ }^{d} R_{\mathrm{BB}}$ is the radius of $\mathrm{BB}$ radiative region. $R_{\mathrm{DBB}}$ is inner radius of the accretion disk. ${ }^{e}$ Photon index of the PL. ${ }^{f}$ Flux ratio (See $\left.\$ 4.2\right)$.

\section{Discussion}

\subsection{Parameter variability}

From the Table 1, we find that the optical depth, $\tau_{\mathrm{BB}}$ is correlated with the luminosity $(\dot{M})$ while $\tau_{\mathrm{DBB}}$ keeps relatively constant. This tendency can be interpreted that the density or the scale of the Compton cloud around NS change more intensively than that of around the accretion disk.

The obtained $R_{\mathrm{BB}}$, assuming the spherical shape, is shown in Table 1 . All of them are much smaller than $10 \mathrm{~km}$ (usual NS radius), and thus we suggest that the radiative region may be zona- 
tion and sparse on the NS. The $R_{\mathrm{BB}}$ and $T_{\mathrm{BB}}$ are also correlated with the $\dot{M}$ but the $R_{\mathrm{BB}}$ is more variable. This indicate that the radiative region has a greater tendency to respond to the $\dot{M}$ than the thermalized temperature.

The obtained all values of $R_{\mathrm{DBB}}$ are also relatively small. We suggest two possibility of this as follows. The first is that the naked cool disk exist and its emission is below the observed range, which results in underestimation of $R_{\mathrm{DBB}}$ (i.e., Comp-DBB is partial radiation from the accretion disk). The second is that the cool disk exist close to the innermost stable circular orbit (e.g., [11]).

\subsection{Comparison of the Radiative efficiency to the super massive black-hole (SMBH), Sgr A*}

In the case of $\mathrm{Sgr}^{*}$, mass accretion rate was estimated $\sim 10^{-8} M_{\odot} / \mathrm{yr}$ (e.g., [1]). If gas accretes at this rate and releases $10 \%$ of energy as the emission, the expected luminosity would be $\sim 10^{38} \mathrm{erg} / \mathrm{s}$. Since the bolometric luminosity of Sgr A* is $\lesssim 10^{37} \mathrm{erg} / \mathrm{s}$ corresponding to $\sim 10^{-6}$ $L_{\text {Edd }}$ (e.g, [12]), the radiative efficiency at the cool or ADAF region of the accretion disk should be $\sim 10^{-2}$ or less. Table 1 shows the flux ratio (FR; flux(Comp-DBB) / flux(Comp-BB) or flux(PL) / flux (Comp-BB)) of $4 \mathrm{U} 1700+24$. Comparing the situation of the NS-LMXB and the SMBH, the Comp-BB and the Comp-DBB (or PL) of the NS-LMXB may correspond to the advected energy to SMBH center and the emitted energy at the accretion disk, respectively. The obtained FR of $4 \mathrm{U}$ $1700+24$ is $\gtrsim 0.1$, indicating that the radiative efficiency in the cool disk or ADAF region of $4 \mathrm{U}$ $1700+24$ is $\sim 10$ times larger than that of Sgr A*. Speculatively, the cause of this difference might be accretion mechanism (Bondie accretion for Sgr A* and wind accretion for $4 \mathrm{U} 1700+24$ ).

\section{References}

[1] D. K. Aitken, J. Greaves, A. Chrysostomou, T. Jenness, W. Holland, J. H. Hough, D. Pierce-Price, and J. Richer, 2000, ApJ, 534, L173

[2] K. Asai, T. Dotani, R. Hoshi, Y. Tanaka, C. R. Robinson, and K. Terada, 1998, PASJ, 50, 611

[3] J. M. Dickey, and F. J. Lockman, 1990, ARA\&A, 28, 215

[4] D. K. Galloway, J. L. Sokoloski, and S. J. Kenyon, 2002, ApJ,580, 1065

[5] M. Gierliński, and C. Done, 2002, MNRAS, 337, 1373

[6] A. K. H. Kong, J. E. McClintock, M. R. Garcia, S. S. Murray, and D. Barret, 2002, ApJ, 570, 277

[7] Q. Z. Liu, J. van Paradijs, and E. P. J. van den Heuvel, 2007, A\&A, 469, 807

[8] Q. Z. Liu, J. van Paradijs, and E. P. J. van den Heuvel, 2006, A\&A, 455, 1165

[9] N. Masetti et. al. 2002, A\&A, 382, 104

[10] J. E. McClintock, and R. A. Remillard, 2006, Black hole binaries (Compact stellar X-ray sources), $157-213$

[11] J. M. Miller, J. Homan, D. Steeghs, M. Rupen, R. W. Hunstead, R. Wijnands, P. A. Charles, and A. C. Fabian, 2006, ApJ, 653, 525

[12] R. Narayan, R. Mahadevan, J. E. Grindlay, R. G. Popham, and C. Gammie, 1998, ApJ, 492, 554

[13] R. Narayan, J. E. McClintock, and I. Yi, 1996, ApJ, 457, 821

[14] J. Poutanen, and R. Svensson, 1996, ApJ, 470, 249

[15] A. Tiengo, D. K. Galloway, T. di Salvo, M. Méndez, J. M. Miller, J. L. Sokoloski, and M. van der Klis, 2005, A\&A, 441, 283 